\title{
Accuracy of Round Meter Gates for On-Farm Deliveries
}

\author{
Daniel J. Howes, M.ASCE${ }^{1}$; and Charles M. Burt, M.ASCE ${ }^{2}$
}

\begin{abstract}
Recent California legislation requires irrigation water agencies larger than 25,000 acres to measure volumetric water deliveries within specified levels of relative uncertainty. Although the meter gate is one of the most widely used flow measurement devices in California, little investigation has been conducted into the accuracy, limitations, and uncertainties of the rating tables developed over 60 years ago. A meter gate testing facility was constructed at the Cal Poly Irrigation Training and Research Center's Water Resources Facility capable of testing gates up to $0.76 \mathrm{~m}$ ( $30 \mathrm{in}$.). The facility was constructed with gates oriented perpendicular to the supply channel flow to match actual field conditions. Three commonly used gate sizes of $0.30 \mathrm{~m}$ (12 in.), $0.46 \mathrm{~m}$ (18 in.), and $0.61 \mathrm{~m}$ ( 24 in.) were examined under a variety of upstream head, head loss, and gate opening conditions. Based on the limitations, guidelines are included to assist users and lower the uncertainty of these devices. It was found that meter gates can be an accurate flow measurement device if installed and operated correctly according to guidelines provided. Limitations were found: upstream head above the top of the turnout pipe must be maintained equivalent to at least half the pipe diameter, and gate openings less than $25 \%$ open can lead to large uncertainties. Using the new rating tables for the three gates examined, the relative uncertainty is less than \pm 5 to $\pm 7 \%$ at the $95 \%$ confidence level with the new rating tables, as compared with less than $\pm 10 \%$ at a $95 \%$ confidence level using common published tables. Uncertainties are lower than the required estimated $10.7 \%$ instantaneous flow rate uncertainty that will be needed to meet current SB X7-7 requirements.
\end{abstract}

\section{Introduction}

Accurate flow measurement of water from irrigation projects delivered to farms is important for a number of reasons. Farmers use the flow measurement and volumes delivered to know how much water was applied to fields; the amount applied must be known for irrigation scheduling and management. Irrigation projects have been shifting from assessment-based fees to volumetric billing (often there is some combination of both). Irrigation district operators also need good turnout flow measurement to properly operate canals.

In California, Senate Bill X7-7 (SB X7-7) was enacted in November of 2009. Specific regulation for agricultural irrigation water agency turnout flow measurement includes (DWR 2011):

- If there is an existing flow measurement device, the volumetric accuracy must be within $\pm 12 \%$; and

- For new flow measurement devices, the volumetric accuracy must be within a laboratory rated \pm 5 or $\pm 10 \%$ in the field if laboratory ratings are not available.

Because of the vast array of conditions in the field, there is no single hardware solution that will economically meet the SB X7-7 requirements in all agricultural water delivery locations throughout California. In most cases, the regions that can use simple solutions already utilize potentially accurate volumetric flow measurement

\footnotetext{
${ }^{1}$ Assistant Professor, Dept. of BioResource and Agricultural Engineering, Irrigation Training and Research Center, California Polytechnic State Univ., San Luis Obispo, CA 93407 (corresponding author). E-mail: djhowes@calpoly.edu

${ }^{2}$ Chairman, Irrigation Training and Research Center, California Polytechnic State Univ., San Luis Obispo, CA 93407. E-mail: cburt@ calpoly.edu
}

devices such as propeller meters. The challenge is finding solutions for the difficult situations. These include areas with high sediment loads, aquatic weeds, little available head loss (where the water levels upstream and downstream of a turnout gate are similar), and high flow rates.

Volumetric accuracy is defined in the SB X7-7 regulations as the percent error between the measured volume and the actual or true volume. The measurement device provides the measured volume (volumes may be computed from a measured flow rate and the duration of delivery) and the actual volume is determined through laboratory or field testing (DWR 2011).

A second term used in this paper is uncertainty, which is the proper term to use when describing the range of values within which the actual value lies for a stated confidence level. In other words, on the average a measured number may equal 0.625 , which is identical to the true value. However, any single measured value may be different.

In some cases, volumetric measurements are made directly by the meter (e.g., propeller meter with totalizing capability). However, instantaneous flow rate is often measured and volumes are estimated based on the duration of the delivery. Since the instantaneous flow rate $(Q)$ may have only been measured at one or more instances during the duration of the delivery, there is some uncertainty beyond the flow meter uncertainty of the volume computed from the device that will influence the volumetric measurement accuracy.

There are several factors that will influence the combined uncertainty of the volumetric measurement from devices such as meter gates, where volumes are computed based on instantaneous flow measurement and the duration of the water delivery. Flow measurement accuracy is a major component of the volumetric accuracy and was investigated for meter gates (special submerged orifice) in this work. The change in supply channel water level between flow measurement reading events will influence the head on the turnout gate and therefore influence the flow rate. Water level 
variation downstream of the orifice will influence the head loss across the gate, which can change the flow rate. Finally, inaccuracy in determining the correct duration of the irrigation event will influence the computed volumetric accuracy. These are discussed in the "Flow Measurement Errors and Uncertainty" section.

One of the most commonly used farm delivery (i.e., turnout) flow measurement devices in California is a meter gate (ITRC 2000, 2002). Meter gates provide a number of advantages if these devices can meet the volumetric accuracy requirements. A major advantage is that thousands of these devices are already installed; water agencies may not need to invest in new devices. Water quality issues including high sediment loads and aquatic weeds do not cause significant problems, and annual maintenance and calibration costs are low with meter gates.

As will be discussed, rating tables exist for common meter gates. One purpose of this work was to compare existing rating table values for several gate sizes against laboratory evaluations. Another was to provide improved gate discharge equations, if found, and to expand the equations to cover a wider range of configurations. Additionally, there was a need to provide clear rules on the installation and operation of these devices to meet the accuracy requirements in SB X7-7. Finally, new rating tables were developed for two rectangular gate sizes commonly installed as new or replacement gates for irrigation turnout delivery (presented in a companion paper).

\section{Background}

The meter gate is a special type of rated orifice (sluice gate) that generally uses a round gate to control water flowing into a round pipeline. Meter gates are submerged and the downstream head is typically measured $0.30 \mathrm{~m}$ (12 in.) downstream of the back face of the gate through an access hole in the top of the pipeline connected to a stilling well. Irrigation agency operators use rating tables for a particular gate size, with measurements of the head loss $(\Delta H)$ between the supply channel and the downstream water level, and the net gate opening $(y)$ to obtain a flow rate through the gate. Rating table development started around 1918 when Modesto Irrigation District began an investigation into calibrating standard gate designs and installations. Modesto ID selected the Calco Slide Headgate Model 101 as its standard gate.

Calco (California Corrugated Culvert Company, Berkeley, California) was a division of Armco (American Rolling Mill Company); the gates became known as the Armco Model 101. The basic design of the round gate on a round pipe is generally referred to as the Armco-type gate. The Armco Model 101 was acquired by Fresno Valves and Casting, (Selma, California) and is still being manufactured as the Series 6600 Model 101 C. Other, similar round canal turnout gates by other manufacturers include the Waterman Industries (Exeter, California) C-10 canal gate and XCAD (Paul, Idaho) X-GATE W-type. The gates by themselves are not meter gates. It is necessary to properly install the tap and stilling well downstream of the gate as well as identify the zero openings to measure flow rates, as will be discussed.

The original Modesto ID ratings were based on submerged gates with different lengths of pipe downstream (Armco 1949). Since the pipe lengths can vary depending on installation, in the mid-1920s Fresno Irrigation District constructed a facility and began developing rating tables using a standard downstream head measurement of $0.30 \mathrm{~m}$ (12 in.) behind the face of the gate, which was also a Calco (Armco) Model 101 (Fresno Irrigation District 1928). Fresno ID conducted tests for gate sizes from 0.20 to $0.61 \mathrm{~m}$ (8 to 24-in.). These rating tables were published by Armco for the Model 101 meter gate until approximately 1951, when the U.S. Bureau of Reclamation (USBR) completed another set of meter gate ratings for gate sizes ranging from 0.20 to $1.22 \mathrm{~m}$ (8 to 48 in.) (Summers 1951). The reason for the USBR rating table development was that the USBR found errors in the Fresno ID ratings of up to $18 \%$ (Summers 1951).

Since the USBR rating development (Summers 1951), very little work has been conducted to examine the accuracy of Armco-type meter gates. Other researchers have used the data collected during the USBR investigation without examining the accuracy of the original data (Cadena and Magallanez 2005).

Prior recommendations on installation of meter gates can be found in the USBR Water Measurement Manual (USBR 1997) and in the Armco Rating Table booklet (Armco Steel Corporation 1975). In the field, there can be a variety of installations that do not conform to either set of recommendations and may have been a result of confusion from alternative instructions or mistakes. The issues seen in the field may be attributed to differing recommendations. For example, the Armco Rating Table booklet and Summers (1951) recommend that the stilling well tap for the downstream head measurement be placed $0.305 \mathrm{~m}$ (12 in.) behind the face of the turnout gate. However, the USBR Water Measurement Manual and Ball $(1961,1962)$ state a preferred distance of onethird of the turnout pipe diameter downstream. The result is a variety of downstream head measurement locations.

Fig. 1 shows the recommended installation of a meter gate with some modifications to the stilling well and pressure tap recommendations based on the authors' experiences with these devices. The stilling well in Fig. 1 is taller than those shown in the USBR Water Measurement Manual and the Armco rating table booklet, which show the top of the well nearly level with the top of the channel bank. Raising the well above the bank prevents debris from depositing in the well when the channel bank road is being graded.

In addition, the top of the stilling well should be level with the top of the gate frame. This allows the operator to measure down from the top of the gate frame and the top of the stilling well to the water surfaces to obtain the head loss $(\Delta H)$. This is an alternative to the typical meter gate well assemblies that can be purchased from manufacturers and those shown in the literature discussed. It is common to see two wells on the downstream side of the gate with the same top elevations. One well is connected to the top of the pipe as shown in Fig. 1 and the other is connected with a horizontal pipe to the upstream canal. The authors have found that this horizontal pipe plugs easily and is very difficult to clean out with water in the upstream canal. In most cases, the upstream water level does not fluctuate significantly so the stilling well for the upstream reading is not necessary.

The stilling well and tap sizes are usually not specified in published meter gate installation recommendations. A stilling well (inside diameter) to (tap diameter) ratio of no less than 8:1 is recommended to dampen the downstream water level fluctuations.

The typical recommendation for the upstream head above the top of the pipe $\left(H_{1}\right)$ is a minimum of one pipe diameter $(D)$. The USBR manual and Armco booklet recommend not having a head loss $(\Delta H)$ greater than $0.46 \mathrm{~m}$ (18 in.) and the Armco tables do not show values for $\Delta H$ greater than this. An updated set of recommendations will be presented in the "Results and Discussion" and "Application" sections of this paper.

The original USBR testing setup for the meter gate ratings was oriented so that the supply water entered the meter gate straight on (parallel to the meter gate discharge pipe) (Summers 1951). The testing conducted for the work presented here had the gates oriented perpendicular to the supply channel flow, which is common in field installations. 


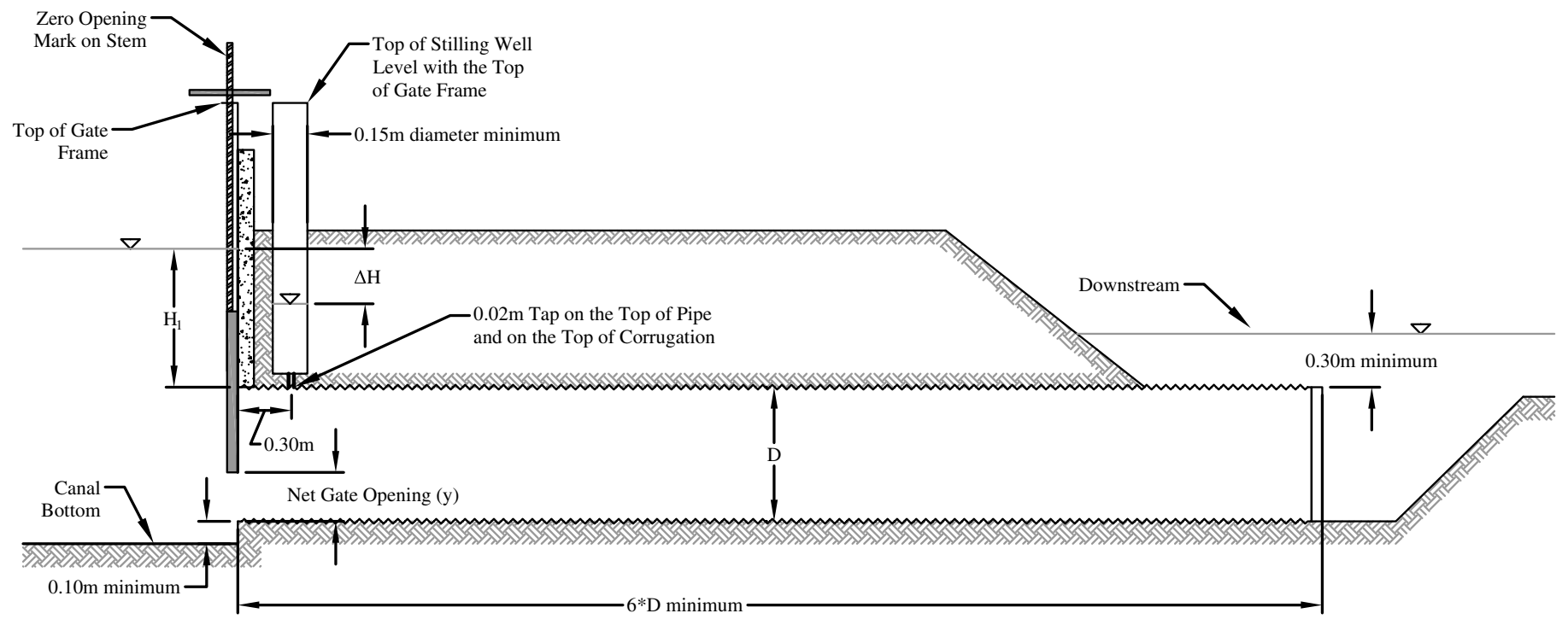

Fig. 1. Improved meter gate installation recommendation

\section{Flow Measurement Errors and Uncertainty}

Several primary factors influence the combined uncertainty of the volumetric measurement from meter gates: flow measurement accuracy, the change in supply channel water level between flow measurement readings, water level variation downstream of the orifice, and inaccuracy in determining the correct duration of the irrigation event. A good discussion on each of these components can be found in Burt and Geer (2012). Since the accuracies of each component are independent, they were combined (Burt and Geer 2012) using the root-sum-of-squares method (Taylor and Kuyatt 1994) to compute the volumetric uncertainty as

$$
U_{V}=100 \times\left[\sqrt{\left(\frac{U_{Q}}{100}\right)^{2}+\left(\frac{U_{H u}}{100}\right)^{2}+\left(\frac{U_{H d}}{100}\right)^{2}+\left(\frac{U_{T}}{100}\right)^{2}}\right]
$$

where $U_{v}$ is the percent (relative) volumetric expanded uncertainty where the resulting value describes the range within which true values lie both in the positive and negative around the measured value with a $95 \%$ confidence level or within two standard deviations (i.e., expanded uncertainty of the volumetric measurement is $\pm U_{v}$ ); $U_{Q}$ is the instantaneous flow measurement accuracy; $U_{H u}$ is the accuracy in flow rate estimated due to variable upstream supply canal water levels; $U_{H d}$ is the accuracy in flow rate estimated due to variable downstream water levels; and $U_{T}$ is the accuracy of the delivery duration estimate. SB X7-7 does not provide a standard coverage factor (number of standard deviations) or confidence level for uncertainty. In the U.S., some organizations base uncertainty and standard error reporting on one standard deviation (67\% confidence level). In this paper, two standard deviations (i.e., $k=2$ and $95 \%$ level of confidence) will be used based on international recommended standards (Taylor and Kuyatt 1994).

An evaluation of upstream supply channel variability for operating turnouts was conducted at San Luis Canal Company (Los Banos, California) during the summer of 2012 (Burt and Geer 2012). Canal water levels were recorded on an hourly basis at 22 sites, collecting data for approximately 90 irrigation deliveries. The channel conditions and structures are typical of many upstream channel distribution systems in the western U.S. with flashboard weir check structures for water level control and submerged orifice turnouts. The results of this evaluation showed that under submerged flow conditions, the uncertainty of flow measurement due to supply channel water level variation $\left(U_{H u}\right)$ was within $\pm 2 \%$ with a $95 \%$ level of confidence.

$U_{H d}$ and $U_{T}$ are influenced by farming practices and irrigation water agency operational rules. Burt and Geer conservatively estimated the expanded $U_{H d}$, or the uncertainty due to change in backpressure on the gate, as $\pm 3 \%$ based on field experience. Additional research is needed to evaluate this uncertainty parameter, and it would depend upon the average elevation change between the supply canal and the farm ditch. The $U_{T}$ of $\pm 4 \%$ was based on a conservative estimate that the difference between actual and recorded duration would be within $\pm 1 h$ within a 24-h delivery period. In many cases irrigation delivery durations are longer than $24 \mathrm{~h}$, which would result in a smaller $U_{T}$ if a $\pm 1 \mathrm{~h}$ error is recorded versus actual duration.

Rearranging Eq. (1) and solving for $U_{Q}$ based on $U_{H u}=2 \%$, $U_{H d}=3 \%, U_{T}=4 \%$, and the $\mathrm{SB} \times 7-7$ requirement of $U_{v}=$ $12 \%$, the relative instantaneous flow measurement uncertainty $\left(U_{Q}\right)$ that can be tolerated is computed to be $\pm 10.7 \%$. The uncertainty of instantaneous flow measurement $\left(U_{Q}\right)$ for meter gates was the focus of the work presented in this paper.

The overall objectives of the study were to check the accuracy of the existing Armco rating tables, provide corrected or more accurate rating tables if necessary, provide laboratory-verified accuracy under a clearly defined set of installation and operational standards, expand the operational range of meter gate rating tables if possible (so that these can be used in a wider range of sites), examine how supply channel velocities influence accuracy, and, when installations do not conform to standards, determine what if any influence this will have on accuracy. The work presented here will cover all topics except for the influence of supply channel velocity, which will be discussed in a future companion paper.

\section{Procedures}

The standard discharge equation for a submerged orifice is

$$
Q=C_{d} A_{o} \sqrt{2 g \Delta H}
$$


where $Q=$ flow rate [cubic meters per second $(\mathrm{CMS})], C_{d}=$ coefficient of discharge, $A_{o}=$ net gate opened area $\left(\mathrm{m}^{2}\right), g=$ gravitational acceleration $\left(9.81 \mathrm{~m} / \mathrm{s}^{2}\right)$, and $\Delta H=$ head loss across the gate (meters). The coefficient of velocity $\left(C_{v}\right)$ has been neglected since the velocity of approach is close to zero because these gates are typically installed perpendicular to the supply channel velocity streamlines.

The $C_{d}$ value can be computed from Eq. (2) as

$$
C_{d}=\frac{Q}{A_{o} \sqrt{2 g \Delta H}}
$$

As will be discussed, a new meter gate testing facility was constructed. Measurements for $Q, A$, and $H$ will be discussed in the following sections.

\section{Meter Gate Testing Facility}

A meter gate testing facility was constructed at the Cal Poly Irrigation Training and Research Center (ITRC) Water Resources Facility. The new testing facility was added to an existing elevated flume near its upstream end (Fig. 2). Prior to modification, the rectangular flume was $1.21 \mathrm{~m}$ wide by $1.21 \mathrm{~m}$ in height on a 0.002 slope. A portion of the flume wall was raised from 1.21 to $1.83 \mathrm{~m}$ for this testing. If the recirculation pump is used, $0.85 \mathrm{~m}^{3} / \mathrm{s}$ can be supplied through the flume. The recirculation pump has a variable frequency drive (VFD) on a 100 horsepower (HP) motor. Flow is measured exiting the pump by a calibrated $0.762 \mathrm{~m}$ McCrometer UltraMag (McCrometer, Hemet, California) magnetic meter on the supply pipeline (not shown in Fig. 2). This water enters a basin at the head of the flume through a $0.762 \mathrm{~m}$ steel pipeline. Flow rates into the flume are controlled by adjusting the VFD to match the target.

As shown in Fig. 2, the meter gates were attached to the flume perpendicular to the flume flow. The meter gate was connected to the side of the flume with a removable steel bulkhead so that the gate frame was attached flush to the side of the steel. The frame and gate protruded slightly into the flow the width of the gate frame as can be found in many field installations. The bottom of the gate was

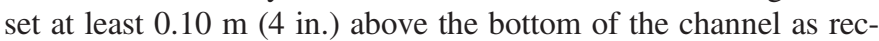
ommended by the USBR. A corrugated discharge pipe, sized to match the gate diameter, connected the gate to the downstream sump as it would be in a typical field installation. The sump on the downstream end of the corrugated pipe had a top elevation equivalent to the top of the flume walls so that a full range of head differentials could be tested.

The Armco and other gate rating tables require the user to measure the net gate stem opening from the zero opening. The zero opening is the point at which the bottom of the gate is level with the bottom of the pipe. To prevent leakage when the gate is closed, the gate plate diameter is larger than the pipe diameter, and the

\section{Top View}

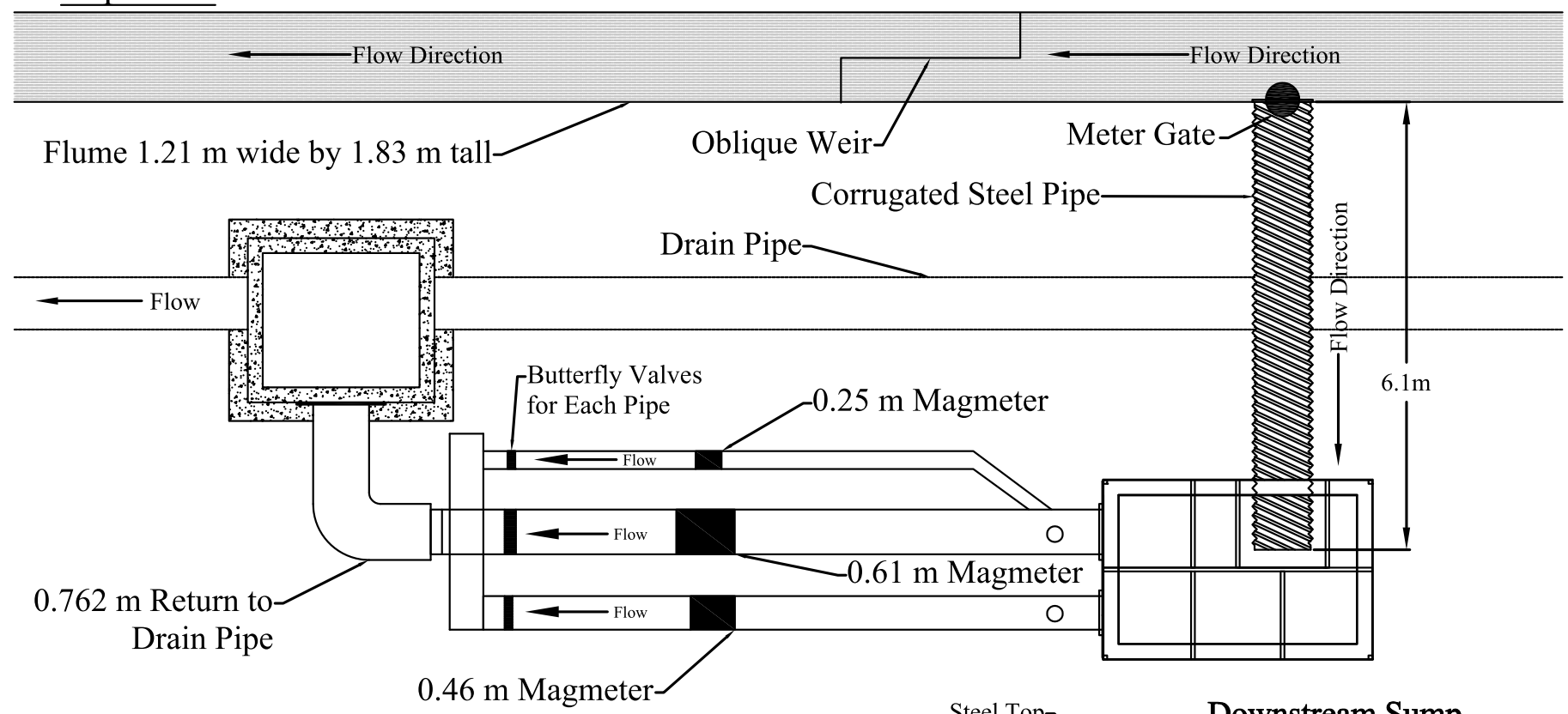

$\underline{\text { Side View }}$

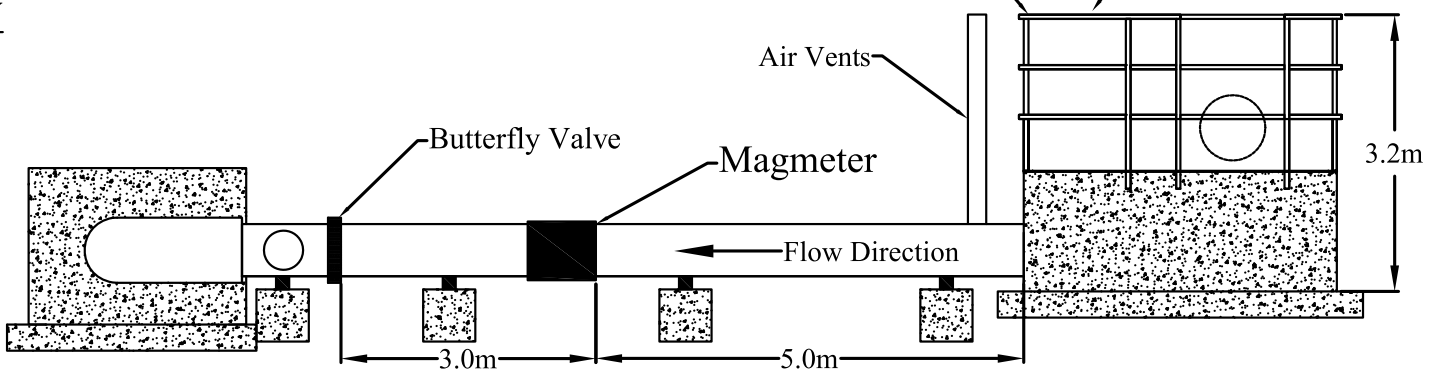

Fig. 2. Top view and side view of the meter gate testing facility at the cal poly ITRC water resources facility 
bottom of the gate seats closed in a position below the inside bottom of the pipe. The gate must open some distance, which varies by gate size and manufacturer, before the zero opening is reached. When a new gate was installed for testing, the stem on each gate was marked to identify the zero opening. A procedure for marking the zero opening is described in the "Application" section.

Three steel discharge pipes were connected to the bottom of the downstream sump (Fig. 2). Each pipe had a calibrated magnetic flow meter (mag meter) with the same inside diameter as the steel pipes; these flow meters were used to determine the flow rate $(Q)$ in Eq. (3). The nominal magnetic (mag) meter sizes are shown in Fig. 2. A discussion on the calibration and operation of these mag meters will be discussed in the next section.

Butterfly valves were installed at the downstream end of each of the mag meter pipes to control the water level in the sump and ensure that the pipe flowed full. These valves were operated manually. The flow leaving the mag meter pipelines entered a manifold where it was discharged into a drain line to be recirculated back to the head of the flume. The drain line ran from an emergency spill at the upper supply sump (to the right of the meter gate in Fig. 2 approximately $18 \mathrm{~m}$ ) down to a sump at the tail end of the flume where the recirculation pump is located.

In the flume, a $3.7 \mathrm{~m}$ long oblique weir was used to control the water level (head) upstream of the meter gate. The weir crest elevation was manually adjusted by adding or removing wood boards (flashboards). All flow passing through the VFD and the $0.762 \mathrm{~m}$ mag meter entered the flume and either passed through the meter gate or went over the weir. The $0.762 \mathrm{~m}$ mag meter was used to measure the flow rate entering the flume so that the velocity of the water in the flume could be known. The results presented in this work utilize very low velocities in the flume to negate the impacts of supply channel velocity on the results and to provide a baseline. The influence of supply channel velocity on meter gate ratings will be discussed in a future publication. Since supply channel velocity will depend on entrance conditions and the channel, it is anticipated that if adjustments are necessary, they would be applied to the baseline ratings developed here.

\section{Flow Rate through the Meter Gate (Q)}

Three magnetic meters were installed downstream of the meter gate to determine the flow rate standard (Q) shown in Eq. (3). The $0.61 \mathrm{~m}$ (24-in.) and $0.46 \mathrm{~m}$ (18-in.) McCrometer UltraMag mag meters and the $0.25 \mathrm{~m}$ (10-in.) Seametrics AG2000 (Seametrics, Kent, Washington) were installed to provide a range of flow testing capabilities. For the results that will be presented here, only one mag meter was used for one test.

Calibration of each meter involved installing it in a pipeline within and parallel to the flume (at different times) prior to the meter gate testing. The meter readings were compared against the flow rate computed from a National Institute of Standards and Technology (NIST) traceable weigh tank at the downstream end. At least nine different flow rates were tested for each meter, and the weigh tank flow rate was compared with the readings from the mag meter. A best-fit linear regression was developed for each gate and used to compute the calibrated flow. The r-squared value for all three calibration equations was greater than 0.999 .

The precalibration average percent error of the $0.61 \mathrm{~m}$ mag meter was $-4.43 \%$. Post-calibration the error was $0.14 \%$ with a root mean squared error (RMSE) of $0.0029 \mathrm{~cm}$ and a coefficient of variation of the RMSE (CVRMSE) of 0.014. The precalibration average percent error for the $0.46 \mathrm{~m}$ mag meter was $-0.67 \%$. Postcalibration the average percent error was $0.07 \%$ with a RMSE of $0.0012 \mathrm{~cm}$ and a CVRMSE of 0.007 . The precalibration average percent error was for the $0.25 \mathrm{~m}$ mag meter was $3.37 \%$. Postcalibration for the $0.25 \mathrm{~m}$ mag meter was $-0.12 \%$ with a RMSE of $0.0017 \mathrm{~cm}$ and a CVRMSE of 0.043 .

Each mag meter had a digital display showing flow rate. Readings were recorded manually during the testing four times for each test after steady state conditions were reached. The calibration equations for each meter gate were applied to the raw flow rates recorded from the digital displays during postprocessing of the data.

\section{Net Gate Opening Area $\left(A_{o}\right)$}

In this study, the actual gate opening area $\left(A_{o}\right)$ was used to compute the $C_{d}$. The original USBR calibration computed $C_{d}$ based on the full pipe area $\left(A_{p}\right)$, not the actual opening area. Therefore, the $C_{d}$ values from this study and the USBR work are not directly comparable. The full pipe $C_{d}$ incorporates the loss across the gate, resulting in $C_{d}$ values that approach zero as the gate opening becomes smaller. The actual gate opening was used here so that differences in actual $C_{d}$ values could be compared between gate openings and different gate sizes. Cadena and Magallanez (2005) computed $C_{d}$ values from the USBR meter gate tests based on an area approximation presented by Hager (1987). However, that area approximation performs poorly at gate openings less than $25 \%$ and greater than $55 \%$, so the $C_{d}$ values computed by Cadena and Magallanez will also not be comparable to those presented in this paper.

An (opening area) to (gate opening position) relationship was derived for a circular gate on a circular pipe. To ensure that the gate seats completely over the pipe, the radius of the gate $\left(R_{g}\right)$ is larger than the radius of the pipe $\left(R_{p}\right)$. The relationship will depend on the gate manufacturer and the gate size. Fig. 3 shows key measurements used to compute the gate opening area.

The following is the relationship between net gate open area $\left(A_{o}\right)$ and net gate opening $(y)$ :

$$
A_{o}=A_{i}-A_{\text {subtracted }}
$$

where

$$
\begin{gathered}
A_{i}=R_{p}^{2} \times \cos ^{-1}\left(\frac{O}{R_{p}}\right)+O \times \sqrt{R_{p}^{2}-O^{2}} \\
A_{\text {subtracted }}=R_{g}^{2} \times \cos ^{-1}\left(\frac{P-O}{R_{g}}\right)+(O-P) \times \sqrt{R_{g}^{2}-O^{2}} \\
P=y+R_{g}-R_{p}
\end{gathered}
$$

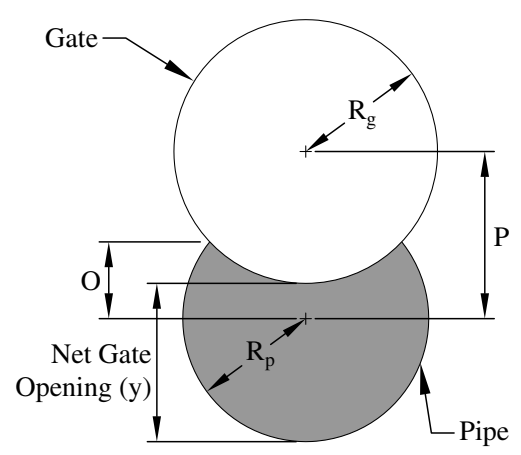

Fig. 3. Meter gate dimensions needed for correct opening area calculation; the gray region is the net gate opening area $\left(A_{o}\right)$ 


$$
O=\frac{R_{p}^{2}-R_{g}^{2}+P^{2}}{2 \times P}
$$

From Fig. 3, $y$ is the net gate opening, $R_{g}$ is the outside radius of the gate, $R_{p}$ is the inside radius of the pipe, $O$ is the distance from the centerline of the pipe to the intersection of the pipe and gate, and $P$ is the distance from the center of the pipe to the center of the gate. In Eqs. (4)-(6), $A_{i}$ is the pipe area at the gate opening if the gate bottom was flat along a geometric chord where the gate intersects the pipe on both sides, and $A_{\text {subtracted }}$ is the area of the circular portion of the gate protruding into the pipe area below this chord. The net gate opening (also referred to as the stem height) is a critical measurement from the bottom of the inside diameter of the pipe to the bottom of the gate. This measurement is often mistaken in the field and as will be discussed, care must be taken to identify the correct zero opening just as the gate breaches the bottom of the pipe.

Eqs. (3)-(7) are equivalent to those used by Skogerboe and Merkely (1996). However, users of that text should be aware of an error in one of the equations presented and refer to an example on the following pages of that chapter to determine the correct equation.

\section{Pressure Head Testing Design}

In order to determine the $\Delta H$ from Eq. (3), the difference in head upstream and downstream of the gate is needed. For this evaluation, multiple locations downstream from the back face of the gate were examined to determine the effect that location of the downstream measurement had on the estimated flow. For a standard meter gate design, the Armco water measurement tables state that the $\Delta H$ is the difference in head between the upstream water level and the water level measured in a stilling well that is connected to the turnout pipeline tapped $0.305 \mathrm{~m}$ (12 in.) downstream from the face of the gate (Armco Steel Corporation 1975, p. 23). This standard location was used for the baseline rating (to compare existing Armco Rating Tables). Fig. 4 shows the locations where downstream head was measured for various readings, including at the $0.305 \mathrm{~m}$ location.

At the top of the closest corrugation to the locations shown in Fig. $4(0.15,0.20,0.305,1.22,2.44,4.88 \mathrm{~m}), 0.019 \mathrm{~m}(3 / 4$ in.) holes were tapped for the head measurements along the pipe. In addition to these locations shown in Fig. 4, head measurements were made for the upstream water level through a $0.019 \mathrm{~m}$ hole in the bulkhead at the same elevation as the top of the corrugated pipe.

Stilling wells were required because of the fluctuation in pressure head in the pipeline; proper sizing is critical, as will be noted in the "Discussion" section. The stilling wells were grouped together for ease of leveling and measurement and were located on the side of the downstream sump wall. The holes were connected to the $0.152 \mathrm{~m}$ (6-in.) PVC stilling wells using $0.016 \mathrm{~m}$ clear flexible plastic hose that was sloped slightly upward from the head measurement location to the stilling wells. Clear plastic hose was used so that air bubbles were visible and could be removed.

The $\Delta H$ was measured directly by using a SMAR-LD301 pressure differential transmitter/transducer (SMAR, Houston, Texas). The LD301 is temperature compensating and the differential range was modified to decrease the output uncertainty to within $\pm 1 \mathrm{~mm}$. The stilling well from the tap connected to the water level in the flume (upstream head) on one side of the differential pressure transducer. The other side of the transducer was connected to a manifold. The manifold connected stilling wells for each downstream head location so that one head measurement location could be isolated with valves and compared with the upstream head for each measurement. The testing personnel would sequentially turn valves on and off and manually take $\Delta H$ readings for each head measurement location.

As redundancy, staff gauges were connected to each stilling well and referenced to the same datum. The staff gauge increments were approximately $1.5 \mathrm{~mm}$. These visual readings were used to manually compute $\Delta H$ to check that the pressure differential transducer was functioning properly. If the $\Delta H$ from the LD301 differed from manual readings by more than $1 \%$, the pressure transducer was zeroed and the test was repeated.

\section{Meter Gate Testing Scenarios}

Testing involved examining a wide array of conditions to examine effects that these conditions have on $C_{d}$. Conditions that were varied included upstream water level in the flume, water level in the downstream sump, gate opening, supply channel velocity, gate size,

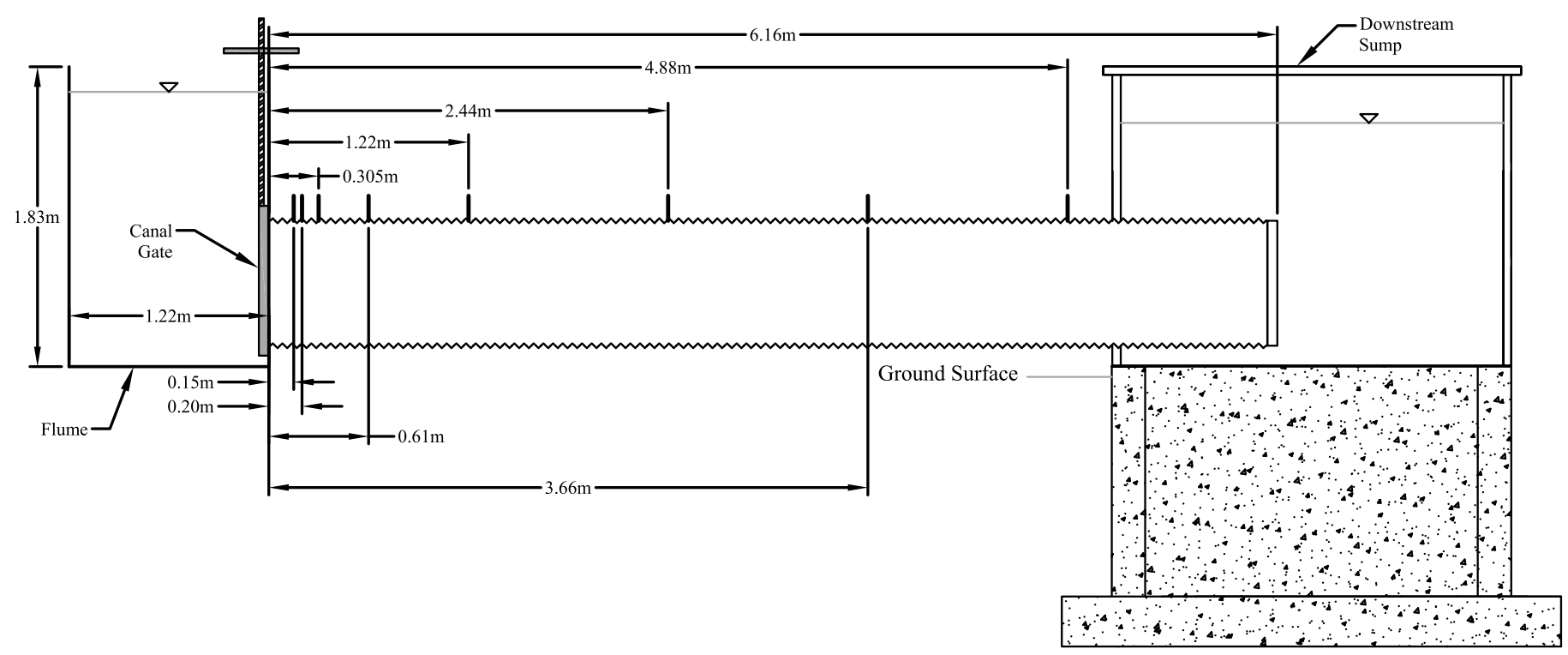

Fig. 4. Side view of the meter gate testing setup and the locations where downstream head measurements were taken 
Table 1. Tests Conducted under Low Supply Channel Velocity for the Meter Gate Testing

\begin{tabular}{|c|c|c|c|c|c|}
\hline Gate type & $\begin{array}{l}\text { Nominal gate } \\
\text { size }(\mathrm{m})\end{array}$ & $\begin{array}{c}\text { Relative } \\
\text { upstream head }\end{array}$ & $\begin{array}{c}\text { Relative } \\
\text { head loss }\end{array}$ & $\begin{array}{l}\text { Upstream head } \\
\left(H_{1}\right) \text { range }(\mathrm{m})\end{array}$ & $\begin{array}{c}\Delta \mathrm{H} \\
\text { range }(\mathrm{m})\end{array}$ \\
\hline Armco & 0.30 & Very low & Very small & $0.251-0.111$ & $0.062-0.041$ \\
\hline Armco & 0.30 & Very low & Small & $0.251-0.111$ & $0.108-0.086$ \\
\hline Armco & 0.30 & Low & Medium & $0.643-0.595$ & $0.182-0.131$ \\
\hline Armco & 0.30 & Middle & Small & $0.643-0.595$ & $0.351-0.305$ \\
\hline Armco & 0.30 & Middle & Medium & $0.643-0.595$ & $0.427-0.378$ \\
\hline Armco & 0.30 & Middle & Large & $0.970-0.845$ & $0.181-0.107$ \\
\hline Armco & 0.30 & High & Small & $0.970-0.845$ & $0.446-0.369$ \\
\hline Armco & 0.30 & High & Medium & $0.970-0.845$ & $0.661-0.613$ \\
\hline Armco & 0.30 & High & Large & $1.235-1.194$ & $0.347-0.310$ \\
\hline Armco & 0.30 & Very high & Medium & $1.235-1.194$ & $0.599-0.563$ \\
\hline Armco & 0.30 & Very high & Large & $1.235-1.194$ & $0.863-0.736$ \\
\hline Armco & 0.46 & Very low & Small & $0.241-0.203$ & $0.070-0.011$ \\
\hline Armco & 0.46 & Low & Medium & $0.489-0.457$ & $0.216-0.156$ \\
\hline Armco & 0.46 & Middle & Small & $0.692-0.597$ & $0.048-0.032$ \\
\hline Armco & 0.46 & Middle & Medium & $0.692-0.597$ & $0.310-0.263$ \\
\hline Armco & 0.46 & Very high & Small & $0.953-0.806$ & $0.185-0.116$ \\
\hline Armco & 0.46 & Very high & Large & $0.953-0.806$ & $0.401-0.358$ \\
\hline Armco & 0.46 & Very high & Very large & $0.953-0.806$ & $0.589-0.538$ \\
\hline Armco & 0.61 & Very low & Small & $0.359-0.283$ & $0.051-0.025$ \\
\hline Armco & 0.61 & Low & Medium & $0.448-0.427$ & $0.212-0.172$ \\
\hline Armco & 0.61 & Middle & Small & $0.694-0.594$ & $0.054-0.029$ \\
\hline Armco & 0.61 & Middle & Medium & $0.694-0.594$ & $0.348-0.260$ \\
\hline Armco & 0.61 & Middle & Large & $0.694-0.594$ & $0.435-0.396$ \\
\hline Armco & 0.61 & High & Small & $0.953-0.841$ & $0.147-0.112$ \\
\hline Armco & 0.61 & High & Large & $0.953-0.841$ & $0.435-0.357$ \\
\hline Armco & 0.61 & High & Very large & $0.953-0.841$ & $0.666-0.540$ \\
\hline
\end{tabular}

and gate type. The results presented here will focus on $\Delta H$ taken at the $0.30 \mathrm{~m}$ (12-in.) downstream head measurement location.

Armco-type (round) gates examined were manufactured and provided by Fresno Valves and Casting. Three common gate sizes were examined, nominally $0.30 \mathrm{~m}$ (12-in.), $0.46 \mathrm{~m}$ (18-in.), and a $0.61 \mathrm{~m}$ (24-in.) Model $101 \mathrm{C}$. These are the same designs as the Armco gates used in the original Fresno Irrigation District and USBR studies.

Table 1 shows the testing range for the different tests conducted as part of the meter gate evaluation under low supply channel velocity. The velocity in the supply channel downstream of the meter gate was always less than $0.2 \mathrm{~m} / \mathrm{s}$ for the results shown here. The goal of this evaluation was to collect data over a wide range of scenarios for each gate size. In large-scale testing, it was not feasible or important to match a predetermined water level or head loss target exactly. Therefore, a target range was attempted for each scenario (e.g., an actual $\Delta H$ of $0.11-0.19 \mathrm{~m}$ would be satisfactory for a test with a target $\Delta H$ of $0.15 \mathrm{~m}$ ) and the results are presented for the actual measured variables.

Upstream head was varied from a classification of Very Low, upward. Very Low would be less than the recommended 1 pipe diameter (corrugated turnout pipe) head above the pipe. Typically this was about 0.5 pipe diameter. The water level for the Low target was typically 1 pipe diameter above the top of the corrugated pipe. The Middle through Very High upstream head ratings were incrementally increased up to the maximum water level that could be safely obtained with the flume wall heights. For the $0.61 \mathrm{~m}$ gate there is no Very High upstream head since the large gate size limited the maximum water level that could be achieved in the flume.

As Table 1 indicates, a variety of head differences were evaluated. The actual head difference (head loss) was varied depending on the upstream head available. With Low upstream head, the limited head available typically resulted in a small head loss.
During each of the tests shown in Table 1, the gate openings were changed. For the $0.30 \mathrm{~m}$ gate, gate openings in increments of $0.025 \mathrm{~m}$ were used from $0.025 \mathrm{~m}$ to fully open. For the 0.46 and $0.61 \mathrm{~m}$ gates, gate openings in increments of $0.05 \mathrm{~m}$ were used from $0.05 \mathrm{~m}$ open to fully open.

Coefficient of discharge $\left(C_{d}\right)$ values were computed for each net gate opening under each scenario in Table 1. Relationships between various testing parameters and the $C_{d}$ will be discussed. The uncertainty of the new $C_{d}$ values as well as the original Armco tables were evaluated by examining the percent error between the actual flow rate measured and that determined using the new rating or Armco table values. Percent error is computed as

$$
E_{Q i}=\frac{Q_{i}-Q}{Q} \times 100
$$

where $E_{Q i}=$ percent error between the estimated flow $\left(Q_{i}\right)$ and the actual flow $(Q)$. The estimated flow $\left(Q_{i}\right)$ was based on the Armco Rating Table $\left(Q_{\text {Armco }}\right)$ and new $C_{d}$ values developed from this work $\left(Q_{\text {improved }}\right)$. The instantaneous flow measurement relative expanded uncertainty (95\% confidence level) was developed based on multiple independent tests with the same gate at each gate opening for the existing Armco tables and the flow rate using $C_{d}$ values from this study. Standard uncertainty of the meter gate $\left(U_{Q}\right)$ was computed as the standard deviation of the error $\left(Q_{i}-Q\right)$ at each gate opening. A coverage factor of $\mathrm{k}=2$ (i.e., \pm 2 standard deviations) was applied for the expanded uncertainty to the $95 \%$ confidence level $\left(U_{Q \_95}\right)$ as

$$
U_{Q \_95}=2 U
$$

The relative expanded uncertainty $\left(R U_{95}\right)$ was computed as

$$
R U_{95}=\frac{U_{Q_{-} 95}}{Q_{\text {mean }}}
$$


where $Q_{\text {mean }}$ is the mean flow rate for the tests for that gate opening. More discussion on the methods used can be found in a number of references (Taylor and Kuyatt 1994; USBR 1997; Lozano et al. 2009).

Values from hardcopy Armco tables (Armco Steel Corporation 1975) provided by Fresno Valves and Casting were entered into a spreadsheet. $Q_{\text {Armco }}$ was determined for each net gate opening and $\Delta H$ by linear interpolation between the two closest $\Delta H$ values for each net gate opening.

\section{Results and Discussion}

One of the primary reasons for testing the same gates under a variety of conditions, even those outside of the ranges shown in the Armco flow measurement tables, is to examine the limitations for the accurate use of meter gates. Potential relationships exist between $\Delta H, H_{1}$, Reynolds number in the turnout pipe $\left(\mathrm{R}_{\text {pipe }}\right)$, and fraction of net gate opening $\left(A_{o} / A_{p}\right)$.

The $C_{d}$ computed for all scenarios is shown in Figs. 5(a-c) for each of the three Armco-type gate sizes examined related to gate opening area. All gate sizes show variability in $C_{d}$ values at the low gate openings. This was also found by Summers (1951) with round gates, and others have reported greater uncertainty at smaller gate openings with rectangular orifice experiments (Lozano et al. 2009).

Fig. 5(a) (0.30 m gate) also shows significant variability in $C_{d}$ values at different fractions of gate opening areas. This variability can be attributed to the Low and Very Low upstream head $\left(H_{1}\right)$ conditions where the upstream head was less than 0.5 times the pipe diameter. However, the 0.46 and $0.61 \mathrm{~m}$ gates performed well for upstream heads as low as 0.5 times the pipe diameter. The Tests Not Excluded in Figs. 5(a-c) represent $C_{d}$ values without upstream head values below 0.5 times the pipe diameter above the top of the pipe for the $0.30 \mathrm{~m}$ gate and gate openings below $20 \%$ for all gates. Additionally, several of the $C_{d}$ values in Fig. 5(a) (0.30 m gate) at gates openings of $30 \%$ not associated with Very Low upstream head were above 1.0. While this is theoretically not possible since there must be energy loss, there are several possible reasons for the inconsistency. Gate leakage is one possibility since the leakage would be a higher percentage of the total flow at the lower gate opening. Measurement error is another possibility. Finally, Eqs. (2) and (3) assume hydrostatic conditions at the upstream and downstream measurement locations. However, these conditions may not necessarily be assumed at the $0.305 \mathrm{~m}$ pressure tap location, specifically at the lower gate opening when the velocity in the vertical direction may be significant close to the gate. This will be investigated in future work. At this point measurement error will be assumed and the values above 1.0 have been removed from further analysis.

Multiple regression analysis was examined on the non-excluded data relating $A_{o} / A_{p}, H_{1} / D$ and $\Delta H / H_{1}$ to $C_{d}$. Using the model

$$
\begin{aligned}
\hat{C}_{d}= & \beta_{5}\left(\frac{A_{o}}{A_{p}}\right)^{3}+\beta_{4}\left(\frac{A_{o}}{A_{p}}\right)^{2}+\beta_{3}\left(\frac{A_{o}}{A_{p}}\right)+\beta_{2}\left(\frac{H_{1}}{D}\right) \\
& +\beta_{1}\left(\frac{\Delta H}{H_{1}}\right)+\beta_{0}
\end{aligned}
$$

where $\hat{\mathrm{C}}_{d}$ is the predicted discharge coefficient, $\beta_{0}$ through $\beta_{5}$ are the regression coefficients, and other variables have been previously defined. The assumptions required for the multiple regression, normality, homoscedasticity, and independence of the errors, was confirmed by residual analysis. The $R_{\text {pipe }}$ was not included in this evaluation since it is highly correlated to $A_{o} / A_{p}$. Results (regression coefficients and corresponding $\mathrm{P}$ values) of the multiple regressions for each gate size tested are shown in Table 2. It can be concluded that $A_{o} / A_{p}$ (for all three gates) and $H_{1} / D$ (for the two larger gates) have some influence on $C_{d}$, while statistically, $\Delta H / H_{1}$ does not affect $C_{d}$. We do not recommend using this multiple regression to compute the $C_{d}$ values because the $H_{1} / D$ and $A_{o} / A_{p}$ values that may be used could be outside of the values used in the multiple regression. Alternative recommendations for determining $C_{d}$ values will be discussed.

Relative upstream head $\left(H_{1} / D\right.$ or upstream head above the pipe divided by the pipe diameter) did have some influence on $C_{d}$ (Table 2) for the two larger gates although the coefficients are low, indicating the influence is relatively small (2-3\% when $H_{1} / D$ is included using Eq. (12) compared to without). This is represented visually in Fig. 6, which shows a relatively constant $C_{d}$ for the same relative gate opening. As indicated in Fig. 5, the lower gate openings generally had higher $C_{d}$ values. Fig. 6 shows that the upstream head above the top of the pipe $\left(H_{1}\right)$ as low as 0.5 times the pipe diameter performed similar to higher heads at and above the recommended minimum head of 1 pipe diameter above the pipe. This indicates that it should be possible to obtain accurate flow measurement in situations where upstream head is less than the recommended 1 pipe diameter, but it should be greater than 0.5 times the pipe diameter.

Fig. 7 shows the relation between relative head loss $\left(\Delta H / H_{1}\right)$ [Figs. 7(a-c)] to $C_{d}$ and Reynolds number [Figs. 7(d-f)] in the corrugated pipe $\left(\mathrm{R}_{\text {pipe }}\right)$ to $C_{d}$. The higher $C_{d}$ values at lower Reynolds numbers can be attributed to the smaller gate openings. Fig. 7

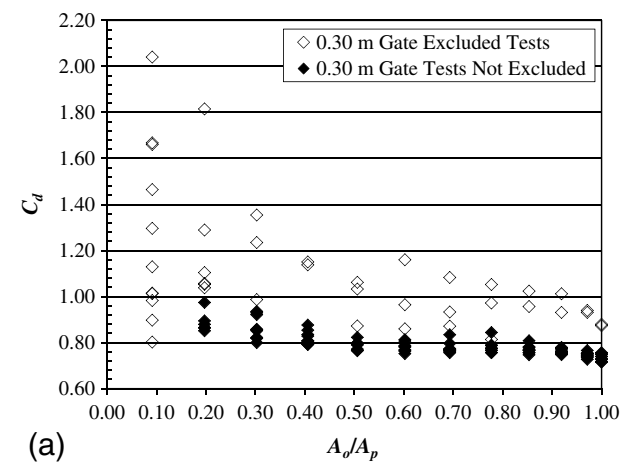

$$
\text { (b) }
$$
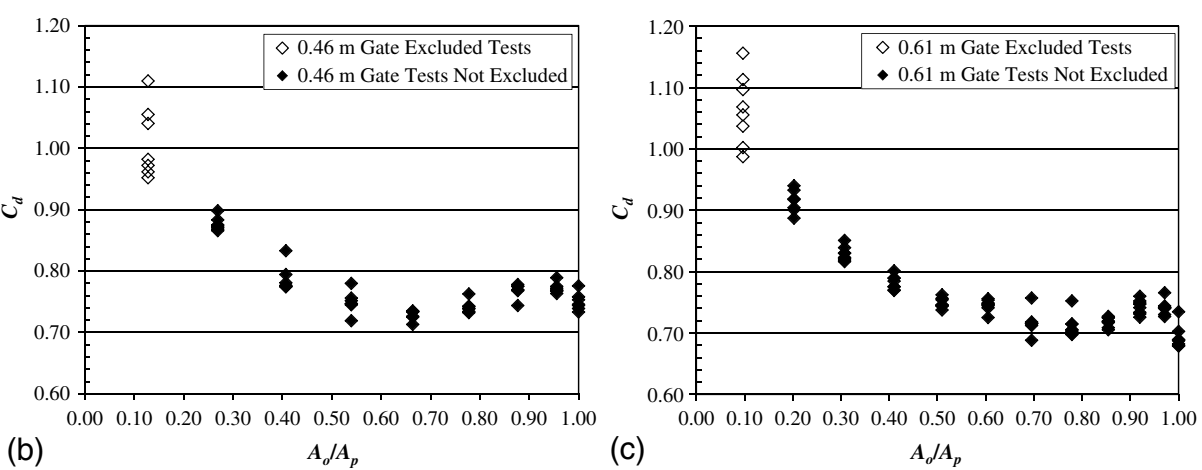

Fig. 5. Coefficient of discharge related to the fraction of gate open area $\left(A_{o} / A_{p}\right)$ for all testing scenarios and excluding data with fraction of net gate openings less than 0.2 , tests with upstream head less than 0.5 times the pipe diameter, and where $C_{d}$ values were greater than 1 ; the $C_{d}$ values are based on measuring the downstream water level at $0.305 \mathrm{~m}$ downstream of the gate for the Armco type: (a) $0.30 \mathrm{~m}$ gate; (b) $0.46 \mathrm{~m}$ gate; (c) $0.61 \mathrm{~m}$ gate 
Table 2. Results of a Multiple Regression Analysis Showing the Test Variables Influencing $C_{d}$ Values for Each Armco-Type Gate Tested

\begin{tabular}{|c|c|c|c|c|c|c|c|}
\hline \multirow[b]{2}{*}{ Predictor } & \multirow[b]{2}{*}{ Coefficient } & \multicolumn{2}{|c|}{$0.30 \mathrm{~m} \mathrm{Armco}^{\mathrm{a}}$} & \multicolumn{2}{|c|}{$0.46 \mathrm{~m} \mathrm{Armco}^{\mathrm{b}}$} & \multicolumn{2}{|c|}{$0.61 \mathrm{~m} \mathrm{Armco}^{\mathrm{c}}$} \\
\hline & & Coefficient & $P$-value & Coefficient & $P$-value & Coefficient & $P$-value \\
\hline$\overline{\left(A_{o} / A_{p}\right)^{3}}$ & $\beta_{5}$ & -0.683 & 0.000 & -1.289 & 0.000 & -0.828 & 0.000 \\
\hline$\left(A_{o} / A_{p}\right)^{2}$ & $\beta_{4}$ & 1.451 & 0.000 & 3.066 & 0.000 & 2.017 & 0.000 \\
\hline$\left(A_{o} / A_{p}\right)$ & $\beta_{3}$ & -1.115 & 0.000 & -2.323 & 0.000 & -1.641 & 0.000 \\
\hline$H_{1} / D^{P}$ & $\beta_{2}$ & 0.001 & 0.089 & -0.013 & 0.004 & -0.018 & 0.005 \\
\hline$\Delta H / H_{1}$ & $\beta_{1}$ & 0.049 & 0.095 & 0.006 & 0.558 & -0.012 & 0.188 \\
\hline Constant & $\beta_{0}$ & 1.041 & 0.000 & 1.324 & 0.000 & 1.194 & 0.000 \\
\hline
\end{tabular}

Note: $P$-values $>0.01$ indicate the variable does not influence $C_{d}$ at an $\alpha$-level $=0.01$.

${ }^{\mathrm{a}} R^{2}=79.6$

${ }^{\mathrm{b}} R^{2}=90.7$.

${ }^{\mathrm{c}} R^{2}=91.8$
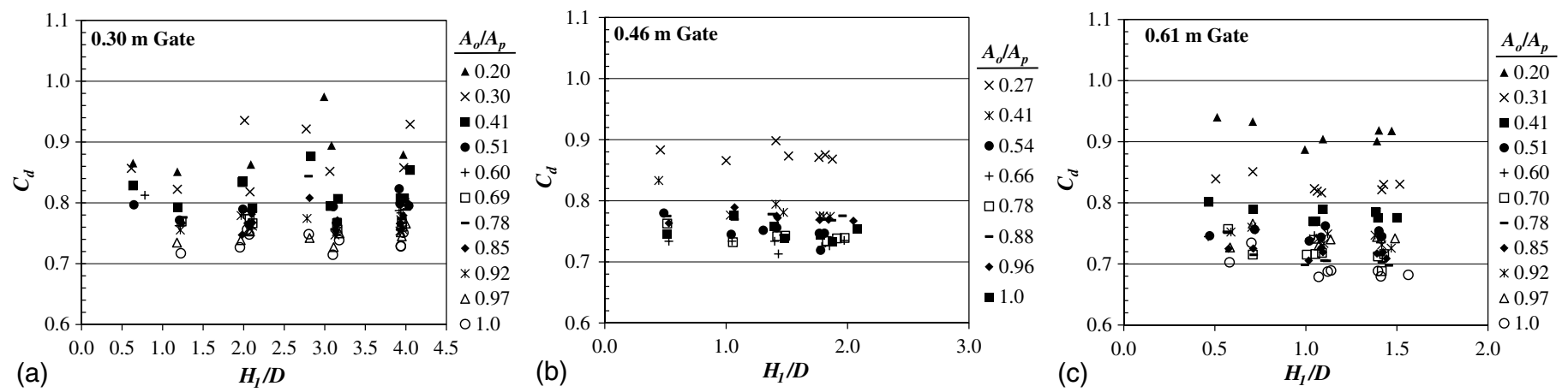

Fig. 6. Coefficient of discharge variation at different relative upstream head $\left(H_{1} / D\right)$ for the fraction of gate openings greater than or equal to 0.2 for the three Armco-type gate sizes examined
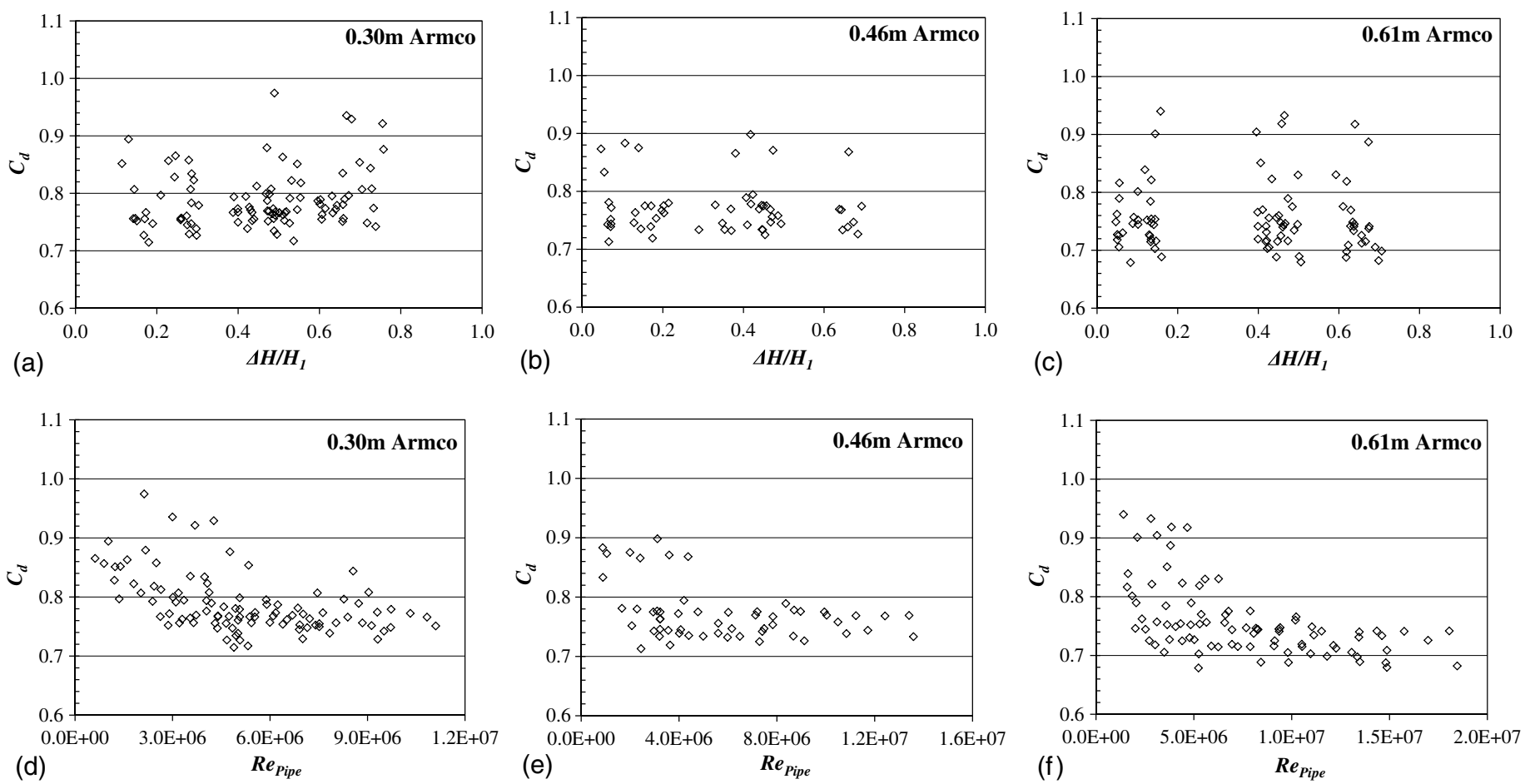

Fig. 7. Coefficient of discharge relationship to relative head loss $\left(\Delta H / H_{1}\right)$ (a-c) and Reynolds number in the turnout pipe ( $\left.\mathrm{R}_{\text {pipe }}\right)$ (d-f) for the three gate sizes 


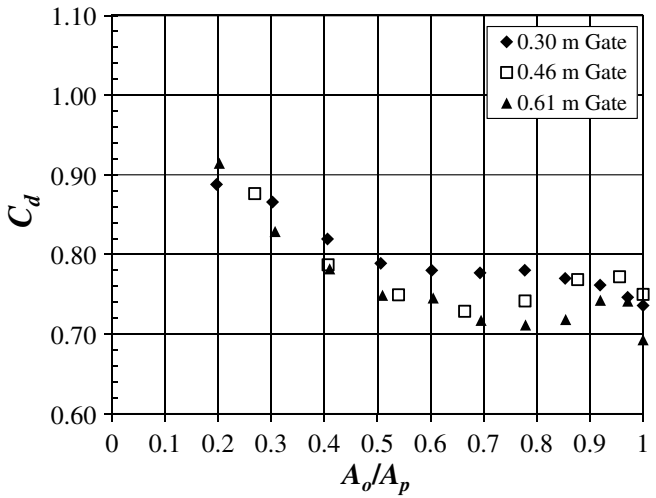

Fig. 8. Average $C_{d}$ computed in this study for the round gates tested in this study by fraction of net gate opening area

indicates that the current recommendation of a maximum $\Delta H$ of $0.46 \mathrm{~m}$ (18 in.) can be exceeded at least with the gates tested in this study. For the $0.30 \mathrm{~m}$ gate (12-in.), $0.46 \mathrm{~m}$ gate (18-in.), and $0.61 \mathrm{~m}$ (24-in.) gate, maximum $\Delta H$ values of $0.86 \mathrm{~m}, 0.59 \mathrm{~m}$, and $0.67 \mathrm{~m}$ were tested, respectively.

During testing $\Delta H$ values were attempted at $\Delta H / H_{1}$ greater than $0.75-0.8$. However, at this point the downstream head in the stilling well would typically drop below the level of the pipe and readings could not be made at the $0.305 \mathrm{~m}$ stilling well (or the closer wells). In field applications, this issue would lead to improper measurements or an inability to take the downstream head measurement.

Fig. 8 shows the average $C_{d}$ by fraction of gate opening area for each gate. Interestingly, the $C_{d}$ values for the 0.46 and $0.61 \mathrm{~m}$ gates are similar when open areas are less than $0.75(75 \%)$. The $C_{d}$ for the $0.30 \mathrm{~m}$ gate is consistently higher than the larger gates when the net open areas are less than $0.75(75 \%)$. At net open areas greater than 0.75 , the $C_{d}$ values seem to consistently stay at approximately 0.75. Figs. 8 and 5 show a dip in $C_{d}$ when the gate reaches full open $\left(A_{o} / A_{p}=1.0\right)$. This is likely due to hydraulic effects as water enters the pipe, with and without gate obstruction, influencing the pressure at the $0.305 \mathrm{~m}$ stilling well.

Of importance to meter gate uses is the uncertainty (accuracy) of the existing rating tables (specifically the Armco rating table booklet) and the $C_{d}$ values computed in this study shown in Fig. 8. Only the three most commonly used gates were investigated. There is a variety of other sizes from 0.20 to $1.22 \mathrm{~m}$ that have rating tables. If good agreement exists between the 0.30, 0.46, and $0.61 \mathrm{~m}$ Armco tables then it might be inferred that similar agreement exists for the other size gates. Fig. 9 shows the average relative error at each gate opening with the relative expanded (95\% confidence level) uncertainty shown bounding the relative error.

As expected, the $0.30 \mathrm{~m}$ gate showed a high level of uncertainty at fractions of gate opening areas $\left(A_{o} / A_{p}\right)$ of less than $40 \%$ (gate opening of $0.10 \mathrm{~m}$ or $4 \mathrm{in}$.). This high variability in $C_{d}$ values shown in Fig. 6 indicates that there was significant variability in the measurements at these low gate openings. At $A_{o} / A_{p}$ of greater than $40 \%$ for the $0.30 \mathrm{~m}$ gate the average relative error was within $7 \%$. The Armco flow tables tended to over-estimate the flow rate for the 0.30 and $0.46 \mathrm{~m}$ gates. This could be caused by the gate arrangement perpendicular to the supply channel flow instead of straight on as they were for the original tests used for the Armco tables. The biased error combined with the expanded uncertainty for the $0.30 \mathrm{~m}$ gate exceeds $+10 \%$ for $A_{o} / A_{i}$ of $50 \%$ and lower. The actual relative errors for $A_{o} / A_{i}$ at 41 and $51 \%$ did not exceed $\pm 10 \%$.

Armco table flow uncertainties ranged from -9 to $+10 \%$ for $A_{o} / A_{p}$ greater than 0.25 for the 0.46 and $0.61 \mathrm{~m}$ gates. This is a good indication that these tables have been providing and will continue to provide good accuracy if the net gate opening area fractions remain greater than $0.25-0.35$ and less than 1.0 (100\% open). In most applications this is the case.

The percent error is significantly reduced with the $C_{d}$ values developed in this study (Table 3). This is to be expected since these $C_{d}$ values were developed from this dataset. Of greater importance is the relative uncertainty, which remains within approximately $\pm 7 \%$ for $A_{o} / A_{p}$ above 0.4 for the $0.30 \mathrm{~m}$ gate and typically within a $\pm 5 \%$ for $A_{o} / A_{p}$ above 0.2 for other gates. It is recommended that users utilize the new $C_{d}$ values developed in this study for the gates tested. For gates that have not been tested, users should utilize the original Armco tables.

\section{Downstream Pressure Tap Location Influence}

Some impact of downstream pressure tap placement will be discussed in this paper since improper placement of the tap relative to the face of the gate is a common occurrence. Fig. 4 shows the downstream pressure tap locations that were investigated. It is common for pressure taps to be placed closer than the recommended 0.305 m location. Fig. 10 shows the $C_{d}$ computed based on the $0.15 \mathrm{~m}$ (6-in.) and $0.20 \mathrm{~m}$ (8-in.) locations compared with the recommended $0.30 \mathrm{~m}$ values from Fig. 8 for the three Armcotype gates. The 0.46 and $0.61 \mathrm{~m}$ gates the $C_{d}$ values are similar for relative fractions of net gate openings less than 0.75 . For the 0.46
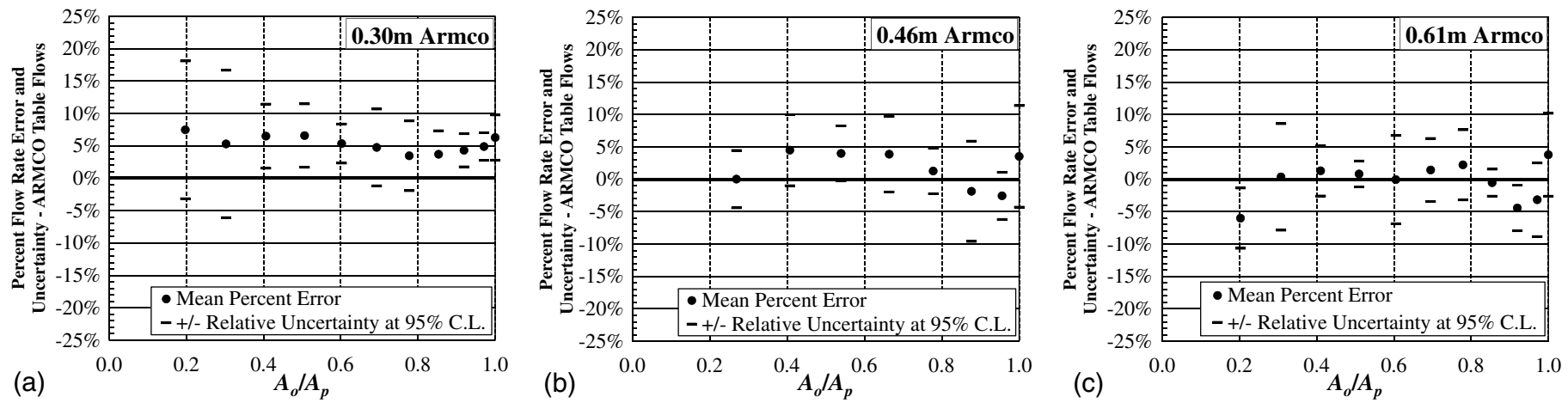

Fig. 9. Average relative error for the three Armco-type gates tested using the original Armco rating tables; relative expanded uncertainty (95\% confidence level) is shown as error bars to indicate the accuracy of the instantaneous flow measurement at different gate opening areas 
Table 3. New $C_{d}$ Values from This Study by Net Gate Opening $(y)$ and Fraction of Net Opening Area $\left(A_{o} / A_{p}\right)$

\begin{tabular}{|c|c|c|c|c|c|c|}
\hline Gate size & $y(\mathrm{~m})$ & $y$ (in.) & $y / y_{o}$ & $A_{o} / A_{p}$ & $C_{d}$ & $\begin{array}{c} \pm \text { Relative } \\
\text { uncertainty }^{\mathrm{a}}(\%)\end{array}$ \\
\hline \multirow{11}{*}{$\begin{array}{l}0.30 \mathrm{~m} \\
(12 \text {-in.) }\end{array}$} & 0.051 & 2 & 0.167 & 0.197 & 0.958 & 9.3 \\
\hline & 0.076 & 3 & 0.250 & 0.303 & 0.878 & 11.4 \\
\hline & 0.102 & 4 & 0.333 & 0.406 & 0.819 & 6.7 \\
\hline & 0.127 & 5 & 0.417 & 0.506 & 0.789 & 4.7 \\
\hline & 0.152 & 6 & 0.500 & 0.602 & 0.780 & 5.1 \\
\hline & 0.178 & 7 & 0.583 & 0.693 & 0.770 & 5.9 \\
\hline & 0.203 & 8 & 0.667 & 0.777 & 0.780 & 6.4 \\
\hline & 0.229 & 9 & 0.750 & 0.853 & 0.770 & 4.3 \\
\hline & 0.254 & 10 & 0.833 & 0.919 & 0.762 & 2.7 \\
\hline & 0.279 & 11 & 0.917 & 0.971 & 0.746 & 3.0 \\
\hline & 0.305 & 12 & 1.000 & 1.000 & 0.736 & 4.0 \\
\hline \multirow{8}{*}{$\begin{array}{l}0.46 \mathrm{~m} \\
(18 \text {-in. })\end{array}$} & 0.102 & 4 & 0.222 & 0.269 & 0.876 & 2.5 \\
\hline & 0.152 & 6 & 0.333 & 0.407 & 0.787 & 5.2 \\
\hline & 0.203 & 8 & 0.444 & 0.540 & 0.749 & 4.8 \\
\hline & 0.254 & 10 & 0.556 & 0.664 & 0.729 & 2.2 \\
\hline & 0.305 & 12 & 0.667 & 0.778 & 0.742 & 2.7 \\
\hline & 0.356 & 14 & 0.778 & 0.877 & 0.769 & 3.1 \\
\hline & 0.406 & 16 & 0.889 & 0.956 & 0.772 & 2.2 \\
\hline & 0.457 & 18 & 1.000 & 1.000 & 0.750 & 3.7 \\
\hline \multirow{11}{*}{$\begin{array}{l}0.61 \mathrm{~m} \\
(24-\mathrm{in} .)\end{array}$} & 0.102 & 4 & 0.167 & 0.202 & 0.915 & 4.0 \\
\hline & 0.152 & 6 & 0.250 & 0.307 & 0.829 & 2.7 \\
\hline & 0.203 & 8 & 0.333 & 0.410 & 0.782 & 2.8 \\
\hline & 0.254 & 10 & 0.417 & 0.510 & 0.749 & 2.1 \\
\hline & 0.305 & 12 & 0.500 & 0.605 & 0.745 & 2.5 \\
\hline & 0.356 & 14 & 0.583 & 0.695 & 0.717 & 5.1 \\
\hline & 0.406 & 16 & 0.667 & 0.779 & 0.711 & 4.8 \\
\hline & 0.457 & 18 & 0.750 & 0.854 & 0.718 & 2.2 \\
\hline & 0.508 & 20 & 0.833 & 0.920 & 0.743 & 3.1 \\
\hline & 0.559 & 22 & 0.917 & 0.971 & 0.741 & 3.1 \\
\hline & 0.610 & 24 & 1.000 & 1.000 & 0.692 & 5.1 \\
\hline
\end{tabular}

${ }^{\text {a }}$ Relative expanded uncertainty at the $95 \%$ confidence level.

and $0.61 \mathrm{~m}$ gates, improper placement should not cause a significant error unless gates are open more than $75 \%$.

In contrast, the $C_{d}$ values vary significantly for $A_{o} / A_{p}$ greater than 0.4 for the $0.30 \mathrm{~m}$ gate. The significantly lower $C_{d}$ values are a result of greater $\Delta H$ measured at the 0.15 and $0.2 \mathrm{~m}$ wells. If a rating table based on the $0.305 \mathrm{~m}$ stilling well location were used with a tap location at 0.15 or $0.2 \mathrm{~m}$, the resulting flow rate would be overestimated. This indicates that if $C_{d}$ values or Armco tables are used for the $0.30 \mathrm{~m}$ gate, the pressure tap location should be moved or a correction factor should be applied to correct for the difference. If stilling wells are located closer than
$0.2 \mathrm{~m}$ to the face of the gate, the flow rates from tables should be multiplied by a correction factor $\left(F_{\text {tap }}\right)$ of 0.95 for gates openings less than or equal to $0.13 \mathrm{~m}(5 \mathrm{in}$.$) , by F_{\text {tap }}=0.89$ for gate openings between 0.13 and $0.23 \mathrm{~m} \mathrm{(} 9 \mathrm{in}$.), and $F_{\text {tap }}=0.86$ for gate openings greater than $0.23 \mathrm{~m}$ (although openings more than $75 \%$ are not recommended).

\section{Application}

As with any flow measurement device, there are constraints and recommendations that must be followed to obtain accurate results. The following guidelines combine some current installation standards, authors' experience, and results found in this study.

1. Traditionally, the upstream head above the top of the turnout pipe $\left(H_{1}\right)$ was recommended to be equivalent to one pipe diameter. However, results in this study show that $H_{1} \geq 0.5 \times D$ provide accurate results. This will increase the number of sites that could potentially utilize meter gates accurately.

2. The range of gate openings should be maintained between 25 and $75 \%$ open (the relationship between gate opening and $A_{o} / A_{p}$ can be found in Table 3). If the stilling well is in the correct location, higher gate openings can be used but should always remain below fully open. For smaller $0.30 \mathrm{~m}$ (12-in.) gates, the minimum opening should be increased to $30-40 \%$. If smaller gate openings are used for only a portion of the season and larger openings for the remainder, the volumetric uncertainty (accuracy) over the season may not be greatly impacted. It is likely that more significant volume will be delivered with the larger gate openings because of the higher flow rates. The volumetric uncertainty caused by the flow rate uncertainty will be proportional to the volume delivered at a specific gate opening. Therefore, the overall instantaneous flow rate uncertainty can be taken as the weighted average uncertainty at gate openings used weighted by the volumes delivered.

3. Sufficient upstream submergence is needed on the downstream end of the turnout pipe. The pipe downstream of the meter gate needs to be full. The water level needs to rise to some measurable level in the downstream stilling well. The downstream submergence should be at least $0.30 \mathrm{~m}$ (12 in.). However, more submergence may be needed so that a $\Delta H$ of approximately $0.76 \mathrm{~m}$ (30 in.) is not exceeded. Previous recommendations limit the head loss to $0.46 \mathrm{~m}$ (18 in.) but head losses greater than this performed well in this study.

4. All rating tables and $C_{d}$ values presented here require knowledge of the net gate opening, as measured by the shaft opening. The zero gate opening must be properly determined and
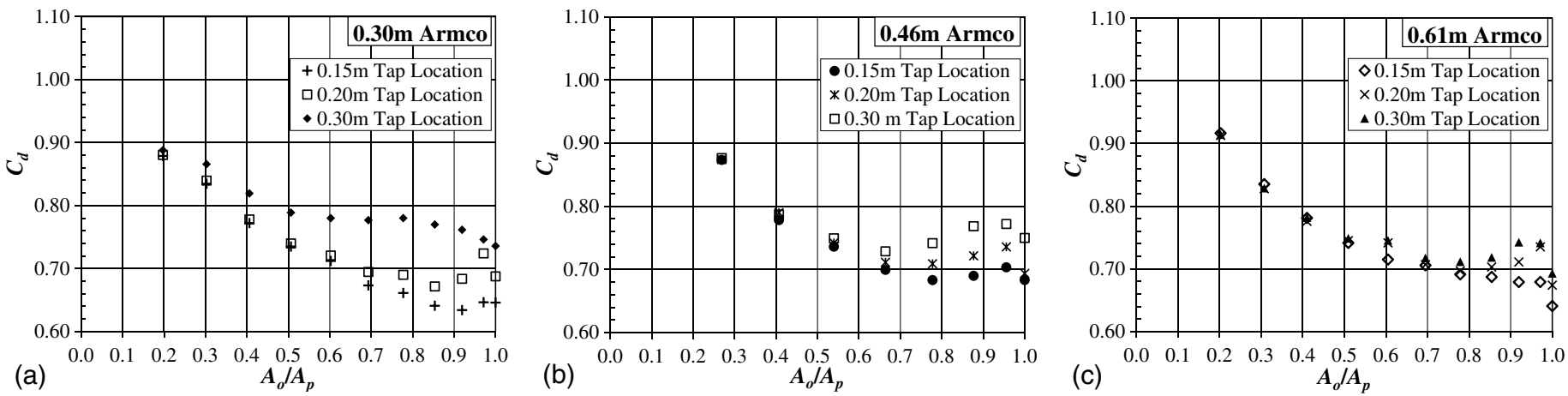

Fig. 10. Effects of downstream tap location on $C_{d}$ at different gate opening fractions 
marked on the gate shaft. This is not a trivial detail. Specific points are:

a. All measurements of gate opening, as well as the initial marking, must be made after the gate stem has been opened (on the upswing). This is because there is some slack or movement between the shaft and the gate itself.

$b$. The gate stem will move up some distance before the gate plate itself reaches the bottom of the pipe. The $C_{d}$ values developed in this study and traditional rating tables depend on knowing the gate opening, not the movement from the gate seating position. The gate must be closed beyond the bottom of the pipe to seal off completely. That sealed position is not the zero position.

c. There must be some specific way to measure the shaft position when the bottom of the gate just barely clears the bottom of the pipe-in other words, when there is a zero opening. This is fairly easy to set and measure if the canal is empty or if a new gate is being installed. The gate is opened until a narrow strip of paper can be inserted between the bottom of the gate and the bottom of the pipe (zero position). If the canal is full, special calipers can be used to determine the actual net gate opening and the zero point on the gate stem can be identified from that.

$d$. The gate stem needs to be marked in a clear manner so that operators know where the zero opening is for the gate when they open the gate. In the field it is often easiest to cut into the stem about $1 \mathrm{~cm}(0.5 \mathrm{in}$.) with a grinder at the top of the gate lift nut. Then the operator should always measure the gate opening on the upswing from the top of the lift nut to the bottom of the notch.

5. The stilling well needs to have sufficient diameter to dampen the turbulence, and so that operators can see into it. The authors recommend a stilling well of $0.15-0.21 \mathrm{~m}(6-8 \mathrm{in}$.) diameter, with a tap hole of about 0.016 or $0.019 \mathrm{~m}(5 / 8$ or $3 / 4$ in.) diameter. The stilling well to tap hole diameters should be greater than $7: 1$.

6. The tap hole must be on the top of the pipe and should be $0.305 \mathrm{~m}$ (12 in.) downstream of the downstream face of the gate. However, the stilling well does not need to be centered over the access hole in the top of the discharge pipe. In general, it is good to have the stilling well close to the gate frame/bulkhead, so that it can be supported.

7. If the stilling well is less than $0.30 \mathrm{~m}$ (12 in.) from the face of the gate for larger gates, the error in measurement will be low if the gates remain less than $75 \%$ open. For $0.30 \mathrm{~m}$ gates and probably smaller, there is a high likelihood of substantial error with different tap locations. It is recommended that the tap location at these sites be moved to the correct location or the correction factor $\left(\mathrm{F}_{\text {tap }}\right)$ should be multiplied by the flow rate obtained from tables based on the $0.305 \mathrm{~m}$ (12-in.) tap location as described.

8. The tap hole should also be on the top of a corrugation if corrugated pipe is used. The closest peak to the $0.305 \mathrm{~m}$ (12-in.) ideal tap location will be sufficient.

9. To simplify the measurement for head difference $(\Delta H)$ use the same datum (elevation) for both measurements. Fig. 1 showed a stilling well with the top correctly placed at the same elevation as the gate frame, and with a proper diameter. The top of the stilling well should be at the same elevation as the top of the gate frame (where the bottom of the lift nut rests). Then the upstream measurement should be taken from the top of the gate frame to the water level. The downstream measurement should be taken from the top of the stilling well to the water level in the well. The $\Delta H$ is the difference between the upstream and downstream measurements from the datum (reference) to the water levels.

10. In many cases having the stilling well top at the same elevation as the top of the gate frame will prevent debris and soil from falling into the well and plugging the tap hole. This can occur during maintenance of the canal bank and road. If the top of the gate frame is still low enough that debris can fall in, a cap should be placed over the well when measurements are not being taken.

11. Volumetric accuracy can be improved from those stated earlier in this paper if:

a. Additional instantaneous flow measurements are taken during the irrigation event. An example would be taking flow measurements every $24 \mathrm{~h}$ at open turnouts even if adjustments are not being made. This will reduce $U_{H u}$ and $U_{H d}$.

b. The time the delivery starts and stops is properly recorded. If operators open and close turnout gates this can be done without additional work.

Table 3 shows the new $C_{d}$ values from this study for the three Armco-type gates examined. It is recommended that these be used for creating new rating tables for these gates. While a best-fit polynomial can be created for each gate [such as that shown in Eq. (12) with coefficients in Table 2], it is more appropriate to interpolate between these values to estimate $C_{d}$ values for other gate openings. Utilizing variables outside of those tested in a regression equation can lead to significant error in the computed $C_{d}\left(\hat{C}_{d}\right)$. Linear interpolation or a more advanced interpolation method can be used. If an advanced interpolation is used the values should be plotted with those reported in this table to ensure that the results conform.

\section{Conclusion}

The meter gate can be an accurate flow measurement device if installed and operated correctly. If the recommendations in the "Application" section of this paper are followed, users of improved rating tables for the $0.30 \mathrm{~m}$ (12-in.), $0.46 \mathrm{~m}$ (18-in.), and $0.61 \mathrm{~m}$ (24-in.) gates can expect the relative uncertainty $\left(U_{Q_{-} \text {new }}\right)$ to be better than \pm 5 to $\pm 7 \%$ at the $95 \%$ confidence level. For other gate sizes the continued use of the Armco flow measurement tables (Armco Steel Corporation 1975) is recommended with an uncertainty $\left(U_{Q \_ \text {Armco }}\right)$ of better than $\pm 10 \%$ at a $95 \%$ confidence level if recommendations are followed. Uncertainties are lower than the required $10.7 \%$ instantaneous flow rate uncertainty discussed previously.

The uncertainty is significantly less using the $C_{d}$ values from this study compared with the original Armco tables. Therefore, new rating tables from $C_{d}$ values developed in this study will be provided to users in digital format by the Irrigation Training and Research Center at California Polytechnic State University, San Luis Obispo. Until the remaining gates can be tested and improved $C_{d}$ values developed, Armco Flow Measurement tables will be made available in digital format, and will be replaced as gates are tested in the future and new tables are developed.

Future evaluations are necessary to develop $C_{d}$ values and expanded tables for other Armco-type gates and gate sizes. Since the Waterman C-10 and XCAD X-Gate have very similar designs as the Fresno Valves and Casting, $101 \mathrm{C}$ gate, it is anticipated that the same rating tables can be used for these gates as well. Additional work is needed to confirm this. The Cal Poly ITRC meter gate testing facility is currently capable of testing gates up to $0.762 \mathrm{~m}$ (30 in.) in size. 
Additionally, research is needed to investigate other uncertainties used to develop the overall volumetric uncertainty. Namely, the change in backpressure or downstream water level variations $\left(U_{H d}\right)$ and potential uncertainty related to durations $\left(U_{T}\right)$ should be examined.

\section{Acknowledgments}

This work was funded by a grant (13-01-005) from the California State University Agricultural Research Institute (CSU ARI). The authors would also like to acknowledge the support provided by San Luis Canal Company, including the donation of gates. The Armco-type gates were provided by Fresno Valves and Castings (Selma, California). The authors would like to acknowledge Evan Geer (former water engineering graduate student) for his help in developing the net open area equations. This work could not have been completed without the hours of work provided by Cal Poly water engineering and irrigation graduate students and construction efforts by ITRC student employees from the BioResource and Agricultural Engineering Department.

\section{Notation}

The following symbols are used in this paper:

$A_{i}=$ pipe area at the gate opening if the gate bottom was flat along a geometric chord where the gate intersects the pipe on both sides;

$A_{o}=$ net gate opening area;

$A_{p}=$ full pipe area;

$A_{\text {subtracted }}=$ area of the circular portion of the gate protruding into the pipe area below a geometric chord;

$C_{d}=$ coefficient of discharge;

$C_{v}=$ coefficient of velocity;

$D=$ pipe diameter;

$E_{Q i}=$ percent error between the estimated flow and the actual flow;

$F_{\text {tap }}=$ correction factor;

$g=$ gravitational acceleration;

$H_{1}=$ upstream head;

$\Delta H=$ change in head;

$O=$ distance from the centerline of the pipe to the intersection of the pipe and gate;

$P=$ distance from the center of the pipe to the center of the gate;

$Q=$ flow rate;

$Q_{\text {Armco }}=$ flow rate from Armco rating table;

$Q_{i}=$ estimated flow rate;

$Q_{\text {improved }}=$ new coefficient of discharge values developed from this work;

$Q_{\text {mean }}=$ mean flow rate;

$\mathrm{R}_{\text {pipe }}=$ Reynolds number in the turnout pipe;

$R_{g}=$ outside radius of the gate;

$R_{p}=$ inside radius of the pipe;

$R U_{95}=$ relative expanded uncertainty;

$U=$ uncertainty;

$U_{H d}=$ accuracy in flow rate estimated due to variable downstream water levels;

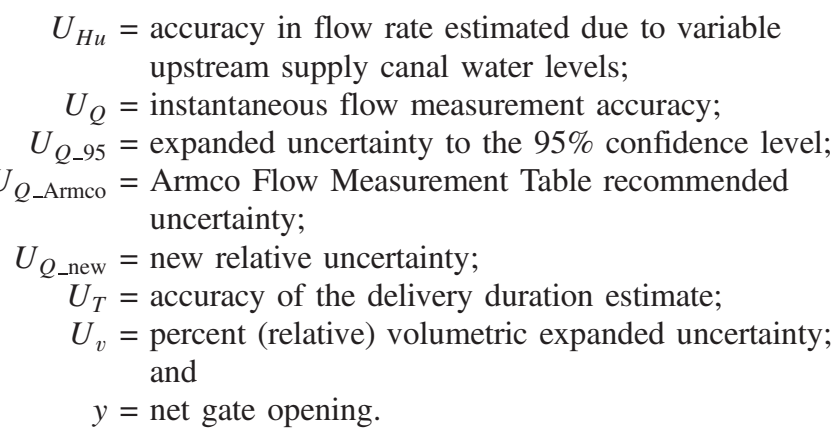

\section{References}

Armco. (1949). "Handbook of water control: For the solution of problems involving the development and utilization of water." Armco Drainage \& Metal Products, Calco Division, Baltimore.

Armco Steel Corporation. (1975). "Water measurement tables: For the Armco metergate." Middleton, $\mathrm{OH}$.

Ball, J. W. (1961). "Flow characteristics and limitations of screw lift vertical metergates." Rep. No. HYD-471, Bureau of Reclamation, Washington, DC.

Ball, J. W. (1962). “Limitations of metergates.” J. Irrig. Drain. Div., 88(4), 23-38.

Burt, C. M., and Geer, E. (2012). "SBx7 flow rate measurement compliance for agricultural irrigation districts." Irrigation Training and Research Center, California Polytechnic State Univ., San Luis Obispo, CA.

Cadena, F., and Magallanez, H. (2005). "Analytical solution for circular gates as flow metering structures." J. Irrig. Drain. Eng., 10.1061/ (ASCE)0733-9437(2005)131:5(451), 451-456.

DWR (Department of Water Resources). (2011). "California code of regulations." Water Conservation Act of 2009: Article 2. Agricultural Water Measurement, California Dept. of Water Resources, Sacramento, CA.

Fresno Irrigation District. (1928). "Methods and devices used in the measurement and regulation of flow to service ditches, together with tables for field use." Fresno, CA.

Hager, W. (1987). "Circular gates in circular and U-shaped channels." J. Irrig. Drain. Eng., 10.1061/(ASCE)0733-9437(1987)113:3(413), 413-419.

ITRC (Irrigation Training and Research Center). (2000). "Status and needs assessment: Survey of irrigation districts-USBR mid-Pacific region." R00-005, California Polytechnic State Univ., San Luis Obispo, CA.

ITRC (Irrigation Training and Research Center). (2002). "Benchmarking of flexibility and needs 2002-Survey of non-federal irrigation districts." R02-007, California Polytechnic State Univ., San Luis Obispo, CA.

Lozano, D., Mateos, L., Merkley, G. P., and Clemmens, A. J. (2009). "Field calibration of submerged sluice gates in irrigation canals." J. Irrig. Drain. Eng., 10.1061/(ASCE)IR.1943-4774.0000085, 763-772.

Skogerboe, G. V., and Merkley, G. P. (1996). Irrigation maintenance and operations learning process, Water Resources Publication, Highlands Ranch, CO, 358.

Summers, J. B. (1951). "Flow characteristics and limitations of Armco meter gates." Rep. No. HYD-314, Bureau of Reclamation, Washington, DC.

Taylor, B. N., and Kuyatt, C. E. (1994). "Guidelines for evaluating and expressing the uncertainty of NIST measurement results." NIST, Gaithersburg, MD, 25.

USBR. (1997). Water measurement manual: A guide to effective water measurement practices for better water management, A Water Resources Technical Publication. U.S. Dept. of the Interior, Bureau of Reclamation, Denver, 485. 